\title{
Community-Based Emergency Care: A Model for Prehospital Care in Remote Canadian Communities
}

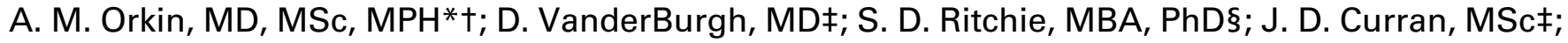 \\ J. Beardy, HBAT
}

\section{INTRODUCTION}

Over 95,000 First Nations people live in 85 remote communities in Canada where federal nursing stations or clinics are the only source of local healthcare. ${ }^{1}$ Most of these communities are without ambulance services; citizens need to board a plane to access an emergency department. Health Canada reports identify first responders as "critical" requirements in remote communities, but untrained laypeople usually deliver on-scene care and transport patients to local health centres. ${ }^{2}$ Despite a \$2.4 billion annual expenditure on Ontario's prehospital care system, approximately 25,000 Ontarians in 29 remote First Nations communities have no formal paramedicine or 911 dispatch services. ${ }^{3}$

Standard ambulance systems, 911 dispatch, and uniformed paramedic professionals have been developed and refined for settings with road access and hospitals, but may not meet first response needs in isolated communities. Addressing unique geographical, cultural, and epidemiological circumstances in remote communities demands a new approach to local medical first response, and the development of systems founded on First Nations self-determination and self-governance of health services. ${ }^{4}$ A community-based approach might answer the urgent call for effective, sustainable, and scalable local care. ${ }^{5,6}$

\section{EMERGENCY CARE IN REMOTE CANADIAN COMMUNITIES}

Remote and isolated First Nations populations in Canada face dramatically elevated morbidity and mortality from mental health and addiction problems, cardiovascular and respiratory diseases, diabetes and obesity, and infectious diseases. ${ }^{1,7}$ These problems all manifest in health emergencies, ranging from mental health crises to myocardial infarction, stroke, diabetic emergencies, severe sepsis, and physical trauma. Remote communities in Northwestern Ontario also face injury rates five to eight times the Canadian average, accounting for $30 \%$ of deaths. ${ }^{8}$

Delivering quality care for these patients requires a strong "chain of survival," with integrated care systems from the place of initial injury or illness to definitive treatment at local clinics or regional hospitals. ${ }^{1,9}$ For most Canadians, that chain of survival begins with bystander first aid and the relatively prompt activation of a formal Emergency Medical Services (EMS).

Health Canada's report on essential services in remote and isolated communities identifies that "CommunityBased First Responders are critical to transport the patient from the community to the nursing stations." ${ }^{2}$ Remote communities in the Nishnawbe Aski Nation (NAN) in Northern Ontario are without formal paramedic services, with the exception of a handful of reserves on the James Bay coast. In a few communities, Canadian Ranger programs and Crisis and Emergency Response Teams deliver fragmented and heterogeneous services. Volunteer teams struggle with burnout, turnover, and inconsistent service. $^{10}$

Patients with urgent medical needs can overwhelm community clinics and nursing staff, who often deliver emergency care beyond their regulated scope of practice while communicating simultaneously with regional consulting physicians and air ambulance providers. ${ }^{1}$ Air ambulance and private aviation services transport patients from nursing stations to regional hospitals. ${ }^{10}$ These evacuations are not infrequent: roughly one in 12 people in the region were transported by air

From the *Dalla Lana School of Public Health, University of Toronto, Toronto, ON; †Rescu, Li Ka Shing Knowledge Institute, St. Michael's Hospital, Toronto, ON; ¥Northern Ontario School of Medicine, Thunder Bay, ON; §Centre for Rural and Northern Health Research, Laurentian University, Sudbury, ON; and the; INishnawbe Aski Nation, Thunder Bay, ON.

Correspondence to: Dr. Aaron Orkin, Dalla Lana School of Public Health, 155 College Street, Toronto, ON, M5T1P8; Email: aorkin@mail.utoronto.ca 
ambulance between January 2012 and September 2013. ${ }^{11}$ In the 2013-2014 fiscal year, Health Canada spent \$175 million on medical transportation for Ontario and Manitoba remote communities, mostly by air ambulance and scheduled flights. During this same period, \$103 million was spent on direct clinical care delivery. ${ }^{1}$

Without formal paramedicine systems in many remote First Nations communities in Canada, bystanders, friends, and family members shoulder the responsibility to transport severely ill and injured patients to local nursing stations and clinics. ${ }^{5,6,10,12}$ The result is a fragile and unpredictable chain of survival. In spite of the occasional heroic success story, these informal systems are an unsafe and unreliable patchwork of community goodwill and clinical near-misses. ${ }^{6,12}$ All are characterized by inadequate training, tragic underfunding, and inexcusable inequity. ${ }^{1,6,10,12}$ Remote communities and their citizens deserve better.

\section{LIGHTS AND SIRENS MAY NOT WORK EVERYWHERE}

The Canadian Association of Emergency Physicians' (CAEP) 1997 position paper on rural, remote, and isolated emergency health care in Canada tasks nursing stations with initial triage of outpatients, definitive care for minor conditions, and stabilization and transfer to hospital where required. ${ }^{13}$ The report asserts that "adequate access to ambulance services should be available in all rural communities," and "regional 911 dispatch by qualified emergency medical dispatchers should be available throughout Canada." ${ }^{14}$ This is the conventional approach to fixing a gap in prehospital care: expand the standard EMS system based on 911 dispatch, professional paramedics, and standardized clinical protocols. While these may be effective strategies in regions with easier access and more resources, conventional approaches are unlikely to meet the needs of remote First Nations communities.

Under previous initiatives and pilot programs, some communities in Ontario's remote north poured funding into ambulances and paramedicine equipment. These were costly, broke down quickly, and required specialized training. ${ }^{10,12}$ Now, old ambulance carcasses litter some community truck dumps. Dust gathers on unused or broken first response equipment in nursing station storage closets. Some communities are without a working defibrillator or serviceable oxygen tank. While accessing and maintaining essential equipment is paramount, the right equipment does not itself guarantee excellent care.

Imposing unmodified standard emergency medicine protocols in settings with limited resources, austere environments, and minimally trained providers will not ensure quality care. In some cases, basic paramedical or first responder standards can be ineffective or dangerous in these settings. Immobilizing a patient on a backboard while waiting hours or days for air ambulance transport in $-40^{\circ} \mathrm{C}$ temperatures may have adverse consequences. Fulfilling recommendations for imaging before reducing a dislocated shoulder is unattainable when there is no hospital available for imaging. Teaching standard first aid courses without modification also has limitations; often, the first step taught is to call 911, but in these settings there is no 911 service, no paramedics, and no local hospital.

Deploying paramedics to fill the emergency care gap in remote communities has the appearance of an appropriate strategy, but conventional paramedicine professionals might not be a good fit for two reasons. First, the Paramedic Association of Canada's professional scopes of practice and Ontario EMS patient care standards presuppose a direct link between paramedicine services, ambulances, emergency equipment, and hospitals, but in remote communities paramedics would provide patient care for longer periods and would interface mostly with nursing stations and air ambulance providers. ${ }^{15}$ Second, mandating and funding paramedicine services in the remote north is unlikely to create sustainable access to service. Health Canada already struggles with significant vacancy rates among rewarding and lucrative nursing positions. ${ }^{1}$ This overwhelming health human resources challenge would likely extend immediately to a paramedicine system reliant on imported professionals.

Through our work with health leaders from remote Northern Ontario communities, we have learned that one-size-fits-all paramedicine protocols and land ambulance service might not meet the needs of patients and providers in remote communities. ${ }^{5}$ CAEP's 1997 position statement identifies that the "principles of emergency medicine do not differ between rural and urban settings... but the method. ... differs significantly." The principle here is that remote communities should have excellent prehospital care. Importing standard ambulance services to remote communities is likely not the right method. Developing local capacity is perhaps a more appropriate strategy. 


\section{COMMUNITY-BASED EMERGENCY CARE}

We propose a different solution based on community workers. Training locals as first responders and educators, instead of focusing on recruiting professionals trained elsewhere, would improve local capacity by giving locals the skills to recognize illness and injury, provide basic treatment, and focus on disease prevention and health promotion. In communities where untrained volunteers are already doing their best to deliver care, community-based programs would support the existing culture of caring and helping behaviour with reasonable remuneration, coordinated leadership, appropriate training, and basic equipment. Local programs could encourage nursing station staff to work with first responders and community health workers. Lay health workers have been shown to deliver transformative maternal-child health and infectious disease interventions in low resource settings—a similar approach might improve emergency care in remote Canadian communities. ${ }^{16}$ In short, a community-based emergency care strategy could reduce unnecessary costs and unnecessary deaths.

Over the past five years, we worked with First Nations leaders to develop Community-Based Emergency Care (CBEC), a new approach to prehospital care in remote communities. ${ }^{5}$ CBEC is a proposed model grounded in the development of a new group of emergency health providers called Community Emergency Health Workers (CEHWs). Supported by a handful of paramedics at the regional level, CEHWs from remote communities would be trained through a customized, comprehensive, and culturally appropriate curriculum, based on their own distinct set of emergency clinical protocols. ${ }^{6}$ Hired in remote communities to deliver essential and timely care, transport patients, and collaborate with local nursing and medical staff, CEHWs would also lead local health promotion and disease prevention programs and train local volunteer first responders. This community-based approach to interdisciplinary healthcare delivery, and the integration of acute care, chronic disease management, and health promotion, extends emerging models for community paramedicine to address health care needs in some of Canada's most underserviced settings. ${ }^{17}$ CEHWs and volunteer responders would form the backbone of a distributed model of community-based emergency care. ${ }^{5}$ CBEC workers would provide a fully integrated model for first response emergency care in these unique geographical and cultural settings, delivering on-scene first response and connecting it with local community care, transport medicine services, and regional advanced care.

Rather than displacing informal systems with standard paramedicine services, CBEC aims to enhance and support existing systems with the training and infrastructure needed to deliver excellent and accessible services. Operated through First Nations governance institutions and delivered by local providers, CBEC contributes to a philosophy of self-determination that has been linked with positive health outcomes and is essential to the cultural vision of First Nations in Canada. ${ }^{4}$ Like similar international initiatives, CBEC would build on a global evidence base demonstrating that local lay health providers can deliver essential culturally appropriate emergency care and transform community health for conditions ranging from physical trauma to mental health. ${ }^{14,18,19,20} \mathrm{CBEC}$ can create jobs, enhance community resilience, improve access to care, and save lives. CBEC offers a new approach to a problem that has been ignored for too long.

\section{CONCLUSIONS}

In remote communities, citizens face stark inequities in access to emergency services, and have identified CBEC as a homegrown approach to delivering care in settings where conventional ambulance services would be ineffective. Local workers can develop the skills to deliver essential features of quality emergency care. In remote communities, excellent evidence-based first response and transport to a local clinic may come in the form of a trained and skilled friend or family member. It is time to invent new approaches to first response and invest in community-based approaches to emergency care.

Keywords: First Response, prehospital care, rural and remote health, community-based health care, Aboriginal health

Acknowledgments: The authors thank the numerous First Nations organizations and communities who supported and contributed to the development of CBEC, and Emma Mew for her assistance in the management of this manuscript.

Competing Interests: Drs. Orkin and VanderBurgh are affiliated with the Remote Health Initiative, a non-profit dedicated to enhancing care in remote settings. The remaining authors declare no conflicts of interest. This commentary received no specific grant from any funding agency, commercial or not-for-profit sectors. Community-Based Emergency Care programming and research has been developed with funding from the Canadian Institutes of Health Research, the Northern 
Ontario Academic Medical Association, and the Indigenous Health Research Development Program.

\section{REFERENCES}

1. Office of the Auditor General of Canada. Access to health services for remote First Nations communities. Report 4, Spring 2015. Available at: http://www.oag-bvg.gc.ca/internet/docs/ parl_oag_201504_04_e.pdf. Archived by WebCite® at: http:// www.webcitation.org/6Yh1LKH5O (accessed May 21, 2015).

2. Health Canada, First Nations and Inuit Health Branch. Basket of Services Working Group Report: Professional Practices Advisory Committee. June 2013.

3. Glazier RH, Gozdyra P, Yeritsyan N. Geographic Access to Primary Care and Hospital Services for Rural and Northern Communities: Report to the Ontario Ministry of Health and Long-Term Care. Toronto: Institute for Clinical Evaluative Sciences; 2011.

4. Reading CL, Wien F. Health Inequalities and Social Determinants of Aboriginal Peoples' Health. National Collaborating Centre for Aboriginal Health; 2009. Available at: http://www. nccah-ccnsa.ca/docs/social\%20determinates/nccah-loppie-wien_ report.pdf. Archived by WebCite® at: http://www.webcitation. org/6YhEcakzP (accessed May 21, 2015).

5. Orkin A, VanderBurgh D, Ritchie S, et al. Community-Based Emergency Care: An Open Report for Nishnawbe Aski Nation. Thunder Bay: Northern Ontario School of Medicine; 2014. Available at: www.nosm.ca/cbec.

6. Orkin A, VanderBurgh D, Born K, et al. Where there is no paramedic: The Sachigo Lake wilderness emergency response education initiative. PLoS Med 2012;9(10):e1001322.

7. Health Canada. First Nations Comparable Health Indicators. Available at: http://www.hc-sc.gc.ca/fniah-spnia/ diseases-maladies/2005-01_health-sante_indicat-eng.php\# mortality. Archived by WebCite ${ }^{\circledR}$ at: http://www. webcitation.org/6Yh9PL49F (accessed May 21, 2015).

8. Whitehead S, Henning B, Johnston J, et al. Developing an Injury Morbidity and Mortality Profile in the Sioux Lookout Zone: 1992-1995. 1996. Project supported by the Canadian Hospitals Injury Reporting and Prevention Program.

9. American Heart Association Emergency Cardiovascular Care Guidelines. Part 12: From Science to Survival Strengthening the Chain of Survival in Every Community. Circulation 2000;102(8 Suppl):I-358-70. Available at: circ. ahajournals.org/cgi/content/full/102/suppl_1/I-358.
10. Ritchie SD, Orkin A, VanderBurgh D, et al. Environmental Scan: Emergency Response \& Training in NAN Communities. Presented at Sioux Lookout, Sudbury, Ontario, October 29-30, 2013. Document available upon request.

11. Personal correspondence, Ornge Air Ambulance, 2014.

12. Born K, Orkin A, VanderBurgh D, et al. 2012. Teaching wilderness first aid in a remote first nations community: the story of the Sachigo Lake Wilderness First Response Education Initiative. Int 7 Circumpolar Health 2012;71. doi: 10.3402/ijch. v71i0.19002.

13. CAEP. Recommendations for the management of rural, remote and isolated emergency bealth care facilities in Canada. Ottawa: Canadian Association of Emergency Physicians; 1997.

14. Kobusingye OC, Hyder AA, Bishai D, et al. Emergency medical systems in low-and middle-income countries: recommendations for action. Bull World Health Organ 2005;83(8):626-31.

15. Paramedic Association of Canada. National Occupational Competency Profile for Paramedics. Available at: http://www. paramedic.ca/uploaded/web/documents/2011-10-31-ApprovedNOCP-English-Master.pdf. Archived at WebCite® at: http:// www.webcitation.org/6c6e4aqgR (accessed October 7, 2015).

16. Lewin S, Munabi-Babigumira S, Glenton C, et al. Lay health workers in primary and community health care for maternal and child health and the management of infectious diseases. Cochrane Database of Systematic Reviews 2010;3: CD004015. doi: 10.1002/14651858.CD004015.pub3.

17. Bigham BL, Kennedy SM, Drennan I, et al. Expanding paramedic scope of practice in the community: a systematic review of the literature. Prehosp Emerg Care 2013;17(3):361-72. doi: $10.3109 / 10903127.2013 .792890$.

18. Jayaraman S, Mabweijano JR, Lipnick MS, et al. First things first: effectiveness and scalability of a basic prehospital trauma care program for lay first-responders in Kampala, Uganda. PLoS One 2009;4(9):e6955.

19. Alaska Community Health Aide Program. Community Health Aide Program Standards and Procedures; 2015. Available at: http://www.akchap.org/resources/chap_library/CHAPCB_ Documents/CHAPCB_Standards_Procedures_Amended_ 2015-01-22.pdf. Archived at Webcite® at: http://www.webci tation.org/6c6kKESTG (accessed October 7, 2015).

20. Chamberlain S, Stolz U, Dreifuss B, et al. Mortality related to acute illness and injury in rural Uganda: Task Shifting to Improve Outcomes. PLoS One 2015;10(4):e0122559. doi:10.1371/journal.pone.0122559. 